\title{
The exact constant in the Rosenthal inequality for random variables with mean zero
}

\section{Citation}

Ibragimov, Rustam and Shaturgun Sharakhmetov. 2002. The exact constant in the Rosenthal inequality for random variables with mean zero. Theory of Probability and Its Applications 46(1): 127-132.

\section{Published Version}

http://dx.doi.org/10.1137/S0040585X97978762

\section{Permanent link}

http://nrs.harvard.edu/urn-3:HUL.InstRepos:2623703

\section{Terms of Use}

This article was downloaded from Harvard University's DASH repository, and is made available under the terms and conditions applicable to Other Posted Material, as set forth at http:// nrs.harvard.edu/urn-3:HUL.InstRepos:dash.current.terms-of-use\#LAA

\section{Share Your Story}

The Harvard community has made this article openly available.

Please share how this access benefits you. Submit a story.

\section{Accessibility}




\title{
THE EXACT CONSTANT IN THE ROSENTHAL INEQUALITY FOR RANDOM VARIABLES WITH MEAN ZERO*
}

\author{
R. IBRAGIMOV ${ }^{\dagger}$ AND SH. SHARAKHMETOV $\ddagger$
}

(Translated by V. A. Vatutin)

Abstract. Let $\xi_{1}, \ldots, \xi_{n}$ be independent random variables with $\mathbf{E} \xi_{i}=0, \mathbf{E}\left|\xi_{i}\right|^{t}<\infty, t>2$, $i=1, \ldots, n$, and let $S_{n}=\sum_{i=1}^{n} \xi_{i}$. In the present paper we prove that the exact constant $\bar{C}(2 m)$ in the Rosenthal inequality

$$
\mathbf{E}\left|S_{n}\right|^{t} \leqq C(t) \max \left(\sum_{i=1}^{n} \mathbf{E}\left|\xi_{i}\right|^{t},\left(\sum_{i=1}^{n} \mathbf{E} \xi_{i}^{2}\right)^{t / 2}\right)
$$

for $t=2 m, m \in \mathbf{N}$, is given by

$$
\bar{C}(2 m)=(2 m) ! \sum_{j=1}^{2 m} \sum_{r=1}^{j} \sum \prod_{k=1}^{r} \frac{\left(m_{k} !\right)^{-j_{k}}}{j_{k} !},
$$

where the inner sum is taken over all natural $m_{1}>m_{2}>\cdots>m_{r}>1$ and $j_{1}, \ldots, j_{r}$ satisfying the conditions $m_{1} j_{1}+\cdots+m_{r} j_{r}=2 m$ and $j_{1}+\cdots+j_{r}=j$. Moreover

$$
\bar{C}(2 m)=\mathbf{E}(\theta-1)^{2 m},
$$

where $\theta$ is a Poisson random variable with parameter 1 .

Key words. Rosenthal inequality, zero mean random variables, moment, Poisson random variable

\section{PII. S0040585X97978762}

Let $\xi_{1}, \ldots, \xi_{n}$ be independent random variables (r.v.'s) with $\mathbf{E} \xi_{i}=0, \mathbf{E}\left|\xi_{i}\right|^{t}<\infty, t>2$, $i=1, \ldots, n$, and $S_{n}=\sum_{i=1}^{n} \xi_{i}$. The following inequality is valid [1]:

$$
\mathbf{E}\left|S_{n}\right|^{t} \leqq C(t) \max \left(\sum_{i=1}^{n} \mathbf{E}\left|\xi_{i}\right|^{t},\left(\sum_{i=1}^{n} \mathbf{E} \xi_{i}^{2}\right)^{t / 2}\right)
$$

where $C(t)$ is a constant depending only on $t$. A number of papers (see, for instance, [2], $[3],[4],[5],[6],[7],[8],[9],[10],[11]$, and [18]), deal with refinements and generalizations of inequality (1) and its analogues. It is shown in [8] that the unimprovable constant $\bar{C}(t)$ in inequality (1) has the order of growth $t / \log t$. Papers [9], [10], and [18] contain the following explicit expressions (being obtained independently) for the exact constant $\bar{C}_{s}(t)$ in (1) for symmetrically distributed r.v.'s: $\bar{C}_{s}(t)=1+2^{t / 2} \Gamma((t+1) / 2) / \sqrt{\pi}, 2<t<4$, $\bar{C}_{s}(t)=\mathbf{E}\left|\theta_{1}-\theta_{2}\right|^{t}, t \geqq 4$, where $\Gamma(a)=\int_{0}^{\infty} x^{a-1} e^{-x} d x$ and $\theta_{1}, \theta_{2}$ are independent Poisson r.v.'s with parameter 0.5 . In [11] the exact value of the constant is found in an analogue of inequality (1) for nonnegative r.v.'s. The same paper also contains some results related

* Received by the editors March 30, 1998; revised March 15, 1999. The first author was supported by a grant from the American Council of Teachers of Russian/American Council for Collaboration in Education and Language Study and by the United States Information Agency.

http://www.siam.org/journals/tvp/46-1/97876.html

${ }^{\dagger}$ Department of Economics, Yale University, P.O. Box 208268, New Haven, CT 06520-8268 (rustam.ibragimov@yale.edu).

‡Tashkent State University, 700098 Tashkent, Uzbekistan (tim001@tseu.silk.glas.org). 
to the exact constants in the inequality $\mathbf{E}\left|S_{n}\right|^{t} \leqq C_{1}(t) \sum_{i=1}^{n} \mathbf{E}\left|\xi_{i}\right|^{t}+C_{2}(t)\left(\sum_{i=1}^{n} \mathbf{E} \xi_{i}^{2}\right)^{t / 2}$, which, like estimate (1), is often called the Rosenthal inequality.

Papers [12], [13], and [14] investigate the problems of finding the exact bounds for the moments of sums of independent r.v.'s in terms of characteristics of particular summands which are closely related to problems of unimprovable constants in moment inequalities. Additional information about basic inequalities for moments of sums of independent r.v.'s as well as a list of relevant papers can be found in [15].

The present paper is close in spirit to the paper of Pinelis and Utev [13] and is devoted to finding the exact constant in a general Rosenthal inequality (assuming no symmetry of the distributions of the r.v.'s in question) for an integer even $t$. The results of the paper were announced in [16].

The following theorem is valid.

Theorem. For $t=2 m, m \in \mathbf{N}$, the exact constant $\bar{C}(2 m)$ in inequality (1) has the form

$$
\bar{C}(2 m)=(2 m) ! \sum_{j=1}^{2 m} \sum_{r=1}^{j} \sum \prod_{k=1}^{r} \frac{\left(m_{k} !\right)^{-j_{k}}}{j_{k} !}
$$

where the internal sum is taken over all natural $m_{1}>m_{2}>\cdots>m_{r}>1$ and $j_{1}, \ldots, j_{r}$, satisfying the conditions $m_{1} j_{1}+\cdots+m_{r} j_{r}=2 m, j_{1}+\cdots+j_{r}=j$. Moreover,

$$
\bar{C}(2 m)=\mathbf{E}(\theta-1)^{2 m}
$$

where $\theta$ is a Poisson random variable with parameter 1.

Remark 1. Clearly, (2) may be rewritten in the following form which looks a bit simpler:

$$
\bar{C}(2 m)=(2 m) ! \sum \prod_{k=1}^{2 m-1} \frac{1}{j_{k} !((k+1) !)^{j_{k}}},
$$

where the sum is taken over all integer-valued solutions of $2 j_{1}+3 j_{2}+\cdots+2 m j_{2 m-1}=2 m$.

Remark 2. It is interesting to note that the exact constant $\bar{C}(2 m)$ in the general Rosenthal inequality does not coincide with the exact constant $\bar{C}_{s}(2 m)$ in the Rosenthal inequality for r.v.'s with symmetric distributions. According to Remark 1 and relation (7.4) in [17], $\bar{C}(2 m)$ is equal to the number of partitions of a set consisting of $2 m$ elements into the parts each of which contains more than one element, while $\bar{C}_{s}(2 m)$ (see [10]) is equal to the number of the partitions of the set into the parts each of which contains an even number of elements.

To demonstrate the theorem we need a number of auxiliary results.

Let $U_{1}, U_{2}$ be independent r.v.'s with distributions $\mathbf{P}\left\{U_{i}=1\right\}=\mathbf{P}\left\{U_{i}=-1\right\}=\frac{1}{2}$, $i=1,2$, let $G$ be a finite positive $\sigma$-additive measure on the $\sigma$-algebra $\mathcal{B}(\mathbf{R})$ of Borel subsets of $\mathbf{R}$, and let $T(G)$ be an r.v. with characteristic function

$$
\mathbf{E} e^{i t T(G)}=\exp \left(\int_{-\infty}^{\infty}\left(e^{i t x}-1\right) d G(x)\right) .
$$

Repeating completely the line of reasoning used in [14] (see also [11]) we establish the following lemma.

LEMMA 1. Let $f: \mathbf{R} \rightarrow \mathbf{R}$ be a continuous nonnegative function satisfying the conditions

$$
\begin{aligned}
f(x)+\mathbf{E} f\left(a_{1} U_{1}+a_{2} U_{2}+x\right) & \geqq \mathbf{E} f\left(a_{1} U_{1}+x\right)+\mathbf{E} f\left(a_{2} U_{1}+x\right), \quad a_{1}, a_{2}, x \in \mathbf{R}, \\
\left|f\left(a_{1}+a_{2}\right)\right| & \leqq C\left(1+\left|f\left(a_{1}\right)\right|\right)\left(1+\left|f\left(a_{2}\right)\right|\right), \quad a_{1}, a_{2} \in \mathbf{R},
\end{aligned}
$$

$(C$ is a constant $)$. If $\int_{-\infty}^{\infty} f(x) d G(x)<\infty$, then

$$
\sup _{n, \xi_{k}} \mathbf{E} f\left(S_{n}\right)=\mathbf{E} f(T(G)),
$$


where sup is taken over all independent zero mean random variables $\xi_{1}, \ldots, \xi_{n}$ such that $\sum_{i=1}^{n} \mathbf{P}\left\{\xi_{i} \in A \backslash\{0\}\right\}=G(A), A \in \mathcal{B}(\mathbf{R})$.

Remark 3. By analogy with Remark 1 in [11] it is easy to show that if $f: \mathbf{R} \rightarrow \mathbf{R}$ is a twice differentiable function, then (4) is a corollary of the convexity of $f^{\prime \prime}(x)$ which implies the $L$-superadditivity of the function $g\left(a_{1}, a_{2}, x\right)=\mathbf{E} f\left(a_{1} U_{1}+a_{2} U_{2}+x\right)$ (see also [14]).

Put $A_{k, n}=A_{k, n}\left(\xi_{1}, \ldots, \xi_{n}\right)=\sum_{i=1}^{n} \mathbf{E} \xi_{i}^{k}, k=1,2, \ldots, 2 m, B_{n}=B_{n}\left(\xi_{1}, \ldots, \xi_{n}\right)=$ $A_{2, n}^{1 / 2}$. By Lemma 1 with $f(x)=x^{2 m}$ and by the Bruno formula for the derivative of the composite function $\exp (g(t)-g(0))$, where $g(t)=\exp \left(\int_{-\infty}^{\infty} e^{i t x} d G(x)\right)$, we obtain that for fixed $A_{k, n}, k=2, \ldots, 2 m$,

$$
\sup \mathbf{E} S_{n}^{2 m}=(2 m) ! \sum_{r=0}^{2 m} \sum \prod_{k=1}^{r} \frac{A_{m_{k}, n}^{j_{k}}\left(m_{k} !\right)^{-j_{k}}}{j_{k} !}
$$

where the internal sum is taken over all natural $m_{1}>m_{2}>\cdots>m_{r}>1$ and $j_{1}, \ldots, j_{r}$, meeting the condition $m_{1} j_{1}+\cdots+m_{r} j_{r}=2 m$.

Let some $A_{2 m}, B, D>0$ be given. Denote $M_{1}\left(m, A_{2 m}, B\right)=\sup _{n, \xi_{k}} \mathbf{E} S_{n}^{2 m}$, where the sup is taken over all independent r.v.'s $\xi_{1}, \ldots, \xi_{n}$ with mean zero and fixed $A_{2 m, n}=A_{2 m}$, $B_{n}=B ; M_{2}\left(m, A_{2 m}, B\right)=\sup _{n, \xi_{k}} \mathbf{E} S_{n}^{2 m}$, where the sup is taken over all independent r.v.'s $\xi_{1}, \ldots, \xi_{n}$ with zero mean and such that $A_{2 m, n} \leqq A_{2 m}, B_{n} \leqq B ; M(m, D)=\sup _{n, \xi_{k}} \mathbf{E} S_{n}^{2 m}$, where the sup is taken over all independent r.v.'s $\xi_{1}, \ldots, \xi_{n}$ with zero mean and fixed $\max \left(A_{2 m, n}, B_{n}^{2 m}\right)=D$.

LEMMA 2. For any integer $s \in(2,2 m)$,

$$
\left|A_{s, n}\right| \leqq\left(A_{2 m, n}^{s-2} B_{n}^{2(2 m-s)}\right)^{1 /(2 m-2)}
$$

and for any $m>1, A_{2 m}>0, B>0$ there exists a sequence of series of random variables $\xi_{n 1}, \ldots, \xi_{n n}, n \geqq n_{0}$, with $\mathbf{E} \xi_{n i}=0, i=1, \ldots, n$, being independent within each series, such that $A_{2 m, n}\left(\xi_{n 1}, \ldots, \xi_{n n}\right)=A_{2 m}, B_{n}\left(\xi_{n 1}, \ldots, \xi_{n n}\right)=B$, and

$$
A_{s, n}\left(\xi_{n 1}, \ldots, \xi_{n n}\right) \longrightarrow\left(A_{2 m}^{s-2} B^{2(2 m-s)}\right)^{1 /(2 m-2)}, \quad n \rightarrow \infty,
$$

for all integer $s \in(2,2 m)$.

Proof. Since $\sum_{i=1}^{n} \mathbf{E}\left|\xi_{i}\right|^{s}=\int_{-\infty}^{\infty}|x|^{s} d G(x)$, inequality (7) follows from the Lyapunov inequality. For $n \geqq\left(B^{2 m} / A_{2 m}\right)^{2 /(m-1)}$ put

$$
\begin{aligned}
a_{n} & =\sqrt{n}\left(\frac{\sqrt{n}+1}{n^{(2 m-1) / 2}+1}\right)^{1 /(2 m-2)}\left(\frac{A_{2 m}}{B^{2}}\right)^{1 /(2 m-2)}, \\
b_{n} & =\left(\frac{\sqrt{n}+1}{n^{(2 m-1) / 2}+1}\right)^{1 /(2 m-2)}\left(\frac{A_{2 m}}{B^{2}}\right)^{1 /(2 m-2)}, \\
p_{n} & =\frac{\left(n^{(2 m-1) / 2}+1\right)^{1 /(m-1)}}{n^{3 / 2}(\sqrt{n}+1)^{m /(m-1)}}\left(\frac{B^{2 m}}{A_{2 m}}\right)^{1 /(m-1)}, \\
q_{n} & =\frac{\left(n^{(2 m-1) / 2}+1\right)^{1 /(m-1)}}{n(\sqrt{n}+1)^{m /(m-1)}}\left(\frac{B^{2 m}}{A_{2 m}}\right)^{1 /(m-1)} .
\end{aligned}
$$

Clearly, $p_{n}+q_{n} \leqq n^{-1 / 2}\left(B^{2 m} / A_{2 m}\right)^{1 /(m-1)} \leqq 1$. Let $\mathbf{P}\left\{\xi_{n i}=a_{n}\right\}=p_{n}, \mathbf{P}\left\{\xi_{n i}=-b_{n}\right\}=q_{n}$, $\mathbf{P}\left\{\xi_{n i}=0\right\}=1-p_{n}-q_{n}, i=1, \ldots, n$. It is not difficult to check that $\mathbf{E} \xi_{n i}=0, i=1, \ldots, n$, $A_{2 m, n}\left(\xi_{n 1}, \ldots, \xi_{n n}\right)=A_{2 m}, B_{n}\left(\xi_{n 1}, \ldots, \xi_{n n}\right)=B$, and for all integer $s \in(2,2 m)$, relation $(8)$ is valid.

Lemma 2 and relation (6) imply the following statement.

Lemma 3. For $A_{2 m}, B>0$

$$
M_{i}\left(m, A_{2 m}, B\right)=(2 m) ! \sum_{j=1}^{2 m}\left(\sum_{r=1}^{j} \sum \prod_{k=1}^{r} \frac{\left(m_{k} !\right)^{-j_{k}}}{j_{k} !}\right)\left(A_{2 m}^{m-j} B^{2 m(j-1)}\right)^{1 /(m-1)}, \quad i=1,2,
$$


where the internal sum is extended over all natural $m_{1}>m_{2}>\cdots>m_{r}>1$ and $j_{1}, \ldots, j_{r}$ satisfying the conditions $m_{1} j_{1}+\cdots+m_{r} j_{r}=2 m, j_{1}+\cdots+j_{r}=j$.

Proof of the theorem. Lemma 3 and the obvious inequality $M_{1}\left(m, D, D^{1 / 2 m}\right) \leqq$ $M(m, D) \leqq M_{2}\left(m, D, D^{1 / 2 m}\right)$ yield

$$
M(m, D)=(2 m) ! \sum_{j=1}^{2 m} \sum_{r=1}^{j} \sum \prod_{k=1}^{r} \frac{\left(m_{k} !\right)^{-j_{k}} D}{j_{k} !},
$$

where the internal sum is taken over all natural $m_{1}>m_{2}>\cdots>m_{r}>1$ and $j_{1}, \ldots, j_{r}$, satisfying the conditions $m_{1} j_{1}+\cdots+m_{r} j_{r}=2 m, j_{1}+\cdots+j_{r}=j$. Hence, taking into account the equality $\bar{C}(2 m)=\sup _{D>0} M(m, D) / D$, we obtain (2).

We show that relations (2) and (3) are equivalent. Let $\theta$ be a Poisson r.v. with parameter 1. Denote by $T_{m}$ the number of partitions of a set consisting of $m$ elements into the parts each of which contains more than one element. According to formula (7.21) in [17] the following relation is valid:

$$
\sum_{m=0}^{\infty} T_{m} \frac{t^{m}}{m !}=e^{e^{t}-t-1} .
$$

Since $\mathbf{E} e^{\theta t}=(1 / e) \sum_{k=0}^{\infty} e^{t k} / k !=e^{e^{t}-1}$, it follows from (9) that $\sum_{m=0}^{\infty} T_{m} t^{m} / m !=$ $\mathbf{E} e^{(\theta-1) t}$ or

$$
\sum_{m=0}^{\infty} T_{m} \frac{t^{m}}{m !}=\sum_{m=0}^{\infty} \mathbf{E}(\theta-1)^{m} \frac{t^{m}}{m !} .
$$

Representation (10) implies $T_{m}=\mathbf{E}(\theta-1)^{m}$. Since $\bar{C}(2 m)=T_{2 m}$ by Remark 2, it follows that $\bar{C}(2 m)=\mathbf{E}(\theta-1)^{2 m}$. The theorem is proved.

A hypothesis. Coincidence of a number of results in [10] and [11] established for r.v.'s having symmetric distributions with known results for r.v.'s with zero mean (see Remark 4 in [10] and Remark 5 in [11]) makes plausible the hypothesis that for $2<t<4$ the exact constant $\bar{C}(t)$ in the general Rosenthal inequality (1) coincides with the exact constant $\bar{C}_{s}(t)$ in inequality (1) for r.v.'s having symmetric distributions. In light of the results of the present paper it seems plausible that $\bar{C}(t)=\mathbf{E}|\theta-1|^{t}$ for $t \geqq 4$, where $\theta$ is a Poisson r.v. with parameter 1 .

Acknowledgment. The authors thank an anonymous referee, whose valuable remarks improved the presentation of the paper in many respects.

\section{REFERENCES}

[1] H. P. Rosenthal, On the subspaces of $L^{p}(p>2)$ spanned by sequences of independent random variables, Israel J. Math., 8 (1970), pp. 273-303.

[2] V. V. Sazonov, On the estimation of moments of sums of independent random variables, Theory Probab. Appl., 19 (1974), pp. 371-374.

[3] S. V. Nagaev and I. F. Pinelis, Some inequalities for the distribution of sums of independent random variables, Theory Probab. Appl., 22 (1977), pp. 248-256.

[4] I. F. Pinelis, Estimates for moments of infinite-dimensional martingales, Math. Notes, 27 (1980), pp. 459-462.

[5] I. F. Pinelis, Optimum bounds for the distributions of martingales in Banach spaces, Ann. Probab., 22 (1994), pp. 1679-1706.

[6] S. V. Nagaev, Some refinements of probabilistic and moment inequalities, Theory Probab. Appl., 42 (1997), pp. 707-713.

[7] S. V. NAGAev, On a new approach to the study of the distribution of a norm of a random element in Hilbert space, in Proceeding of the Fifth Vilnius Conference on Probability Theory and Mathematical Statistics, Mokslas, Vilnius, 1990, pp. 214-226. 
[8] W. B. Johnson, G. Schechtman, And J. Zinn, Best constants in moment inequalities for linear combinations of independent and exchangeable random variables, Ann. Probab., 13 (1985), pp. 234-253.

[9] R. Ibragimov and Sh. Sharakhmetov, On the exact constant in the Rosenthal inequality, in Abstracts of the 4th Fergan International Colloquium in Probability Theory and Mathematical Statistics, Tashkent, 1995, pp. 43-44 (in Russian).

[10] R. Ibragimov and Sh. Sharakhmetov, On an exact constant for the Rosenthal inequality, Theory Probab. Appl., 42 (1997), pp. 294-302.

[11] R. Ibragimov and Sh. Sharakhmetov, Some extremal problems in the moment inequalities, (in print).

[12] Yu. V. Prokhorov, Extremal problems in limit theorems, in Proceedings of the Sixth All-Union Meeting in Probability Theory and Mathematical Statistics, Vilnius, 1962, pp. 77-84 (in Russian).

[13] I. F. Pinelis And S. A. Utev, Estimates of moments of sums of independent random variables, Theory Probab. Appl., 29 (1984), pp. 574-578.

[14] S. A. UTEv, Extremal problems in moment inequalities, in Limit Theorems of Probability Theory, Trudy Inst. Math. 5, "Nauka" Sibirsk. Otdel., Novosibirsk, 1985, pp. 56-75 (in Russian).

[15] G. Peshrir And A. N. ShiRyaev, Khinchin inequalities and a martingale extension of the sphere of their action, Russian Math. Surveys, 50 (1995), pp. 849-904.

[16] R. Ibragimov and Sh. Sharakhmetov, Exact bounds on the moments of symmetric statistics, in Proceedings of 7th Vilnius Conference on Probability Theory and Mathematical Statistics, 22nd European Meeting of Statisticians: Abstracts of Communications, Vilnius, 1998, pp. 243-244.

[17] V. N. SachKov, Combinatorial Methods in Discrete Mathematics, Cambridge University Press, Cambridge, UK, 1996.

[18] T. Figiel, P. Hitczenko, W. B. Johnson, G. Schechtman, And J. Zinn, Extremal properties of Rademacher functions with applications to the Khinchine and Rosenthal inequalities, Trans. Amer. Math. Soc., 349 (1997), pp. 997-1027. 